Ann. Sci. forest., 1972, 29 (4), 403-426.

\title{
DENSITÉ DE SEMIS, EFFETS DE COMPÉTITION, ET VARIABILITÉ INTRASPÉCIFIQUE CHEZ LE DOUGLAS EN PÉPINIÈRE
}

\author{
Y. BIROT \\ avec la collaboration technique de E. PoHoski et Mme D. AuberT \\ Station d'Amélioration des Arbres forestiers \\ Centre national de Recherches forestières, I.N.R.A. \\ Champenoux 54370 Einville
}

\begin{abstract}
RÉSUMÉ
Le problème de la compétition créée par la densité entre les individus d'une population est souvent abordé indépendamment des caractéristiques génétiques de la population étudiée. Il est possible cependant qu'il existe des interactions génotypes $x$ densité. La présente note fait le point d'une expérience destinée à étudier en pépinière la variabilité intraspécifique du comportement avec la densité, de jeunes plants de Douglas issus de provenances différentes. Le test a porté sur trois provenances à trois niveaux de densité $\left(400 / \mathrm{m}^{2}=5 \times 5 \mathrm{~cm}, 100 / \mathrm{m}^{2}=10 \times 10 \mathrm{~cm}, 25 / \mathrm{m}^{2}=20 \times\right.$ $20 \mathrm{~cm})$. Les plants ont été obtenus par semis direct.

Il est possible de mettre en évidence un certain nombre d'effets de la densité sur des caractères phénologiques ou de vigueur. Pour le diamètre, des effets de compétition sont également observés. Ceci conduit à recommander pour les essais de provenance, en pépinière, des densités plus faibles que celles habituellement utilisés. Par contre, il n'est pas possible de montrer l'existence d'interactions génotype $\times$ densité. Compte tenu de l'échantillonnage limité de provenances, on ne peut cependant étendre ce résultat à l'ensemble de l'espèce Douglas.
\end{abstract}

\section{INTRODUCTION}

Le problème de la compétition entre individus est généralement abordé, par le forestier sous l'angle sylvicole, par l'étude de la densité de plantation ou par l'étude des éclaircies. Dans la plupart des expériences de ce type, il n'est pas tenu compte de la variabilité intraspécifique : on admet que le matériel végétal utilisé dans l'expérience est représentatif de l'espèce.

Or, la plupart des espèces forestières présentent une forte variabilité intraspécifique, que révèlent les plantations comparatives de provenances. On peut penser alors que le comportement de provenances vis-à-vis d'une situation de compétition créée par la densité de plantation est susceptible d'être lui-même variable. 
A partir de cette hypothèse, $P$. Bouvarel a conçu et réalisé l'expérience dont les résultats sont présentés ici. Son but est l'étude du comportement vis-à-vis de la densité de plusieurs provenances de Douglas, espèce dont la forte variabilité intraspécifique et la croissance initiale rapide sont connues.

Le dispositif expérimental utilisé ne permet de mesurer les effets de densité ou de compétition qu'à l'intérieur des provenances et non entre les provenances.

La question principale est la suivante : y a-t-il interaction entre provenance et densité de semis?

\section{I. - MATÉRIEL ET MÉTHODES D’ÉTUDE}

\section{1. - Le matériel végétal}

Trois provenances ont été choisies : une provenance artificielle française et deux provenances naturelles américaines; leur localisation est rappelée dans le tableau 1.

TABLEAU 1 - TABLE 1

Localisation des provenances

Location of provenances

\begin{tabular}{|c|c|c|c|c|}
\hline Stations & Latitude & Longitude & $\begin{array}{c}\text { Altitude } \\
\text { m }\end{array}$ & Abréviation \\
\hline $\begin{array}{l}\text { Granite Falls... U.S.A. — Washington ..... } \\
\text { Manning _ Park... Canada-Br-Columbia .. } \\
\text { St-Just-d'Avray... France (Rhône) . . . . . . } \\
\text { (origine inconnue) }\end{array}$ & $\begin{array}{l}48^{\circ} 05 \\
49^{\circ} 15 \\
45^{\circ} 57\end{array}$ & $\begin{array}{r}122^{\circ} 00 \mathrm{~W} \\
121^{\circ} 10 \mathrm{~W} \\
2^{\circ} 06 \mathrm{E}\end{array}$ & $\begin{array}{l}190 \\
720 \\
750\end{array}$ & $\begin{array}{l}\mathrm{GR}-\mathrm{FA} \\
\mathrm{MA}-\mathrm{PA} \\
\mathrm{SJ}-\mathrm{AV}\end{array}$ \\
\hline
\end{tabular}

Le choix des provenances était dicté par :

- la nécessité d'étudier un faible nombre de provenances pour ne pas alourdir le dispositif, - l'intérêt d'observer des provenances aussi diverses que possible. Compte tenu des résultats obtenus antérieurement en plantation comparative, on a choisi une provenance vigoureuse et à débourrement végétatif tardif, Granite Falls, une provenance à croissance initiale faible et précoce, Manning Park. Enfin, la provenance artificielle, St-Just-d'Avray, pouvait mettre en jeu une structure génétique différente.

\section{2. - Le dispositif expérimental}

Trois niveaux de densité ont été retenus selon la progression suivante :

- 25 semis au $\mathrm{m}^{2}$ (écartement $20 \times 20 \mathrm{~cm}$ );

-100 semis au $\mathrm{m}^{2}$ (écartement $10 \times 10 \mathrm{~cm}$ );

- 400 semis au $\mathrm{m}^{2}$ (écartement $5 \times 5 \mathrm{~cm}$ ).

Le dispositif utilisé est un dispositif factoriel $3^{2}$ ( 3 provenances $\times 3$ densités) en blocs complets avec 4 répétitions.

Les parcelles unitaires contiennent un nombre sensiblement identique de semis :

- $5 \times 5: 60$ semis non compris une

$-10 \times 10: 56$ semis ligne périmétrale

$-20 \times 20: 57$ semis) d'isolement

$\mathrm{Si}$ ce dispositif permet de comparer les traitements de façon rigoureuse, on rencontre cependant une difficulté déjà signalée par ILLY et LEMOINE (1970) : le nombre de plants étant sensiblement constant aux différents niveaux de densité, il en résulte que la surface du terrain occupé est beaucoup plus faible aux fortes densités. Dans le cas qu'ils étudiaient, ils obtenaient à ces fortes densités une corrélation positive entre plants voisins due au terrain, et la variance ou la covariance dues à la compétition étaient sous-estimées. 


\section{3.- Le terrain; les travaux}

Le dispositif décrit a été installé à la pépinière du C.N.R.F. à Amance près de Nancy.

Chaque bloc est représenté par une bâche dont la préparation a consisté en un surfaçage avec de la tourbe, les graines étant recouvertes ensuite avec de la tourbe criblée.

Les semis ont été effectués le 30 juin et $1^{\text {er }}$ juillet 1967, à raison de 3 graines prétraitées par emplacement. Le démariage a été réalisé ultérieurement par section au ciseau des plants excédentaires. Au cours de cette opération, une certaine sélection en faveur des plantules les plus vigoureuses a pu jouer.

A la fin de la première année (1967) et de la deuxième année (1968) on a remplacé les plants morts, ainsi qu'en mai 1969, mais cette fois pour un très faible nombre de plants. Les plants regarnis n'ont pas été pris en compte dans les mensurations.

Enfin, les entretiens classiques de désherbage ont été réalisés.

\section{4. - Observations et mensurations}

Les observations et mensurations effectuées ont été résumées dans le tableau 2. Le niveau individuel est toujours conservé.

TABLEAU 2 TABLE 2

Observations et mensurations

Studied characters

\begin{tabular}{|c|c|c|c|}
\hline Caractère & Effectif & Date & Observations \\
\hline 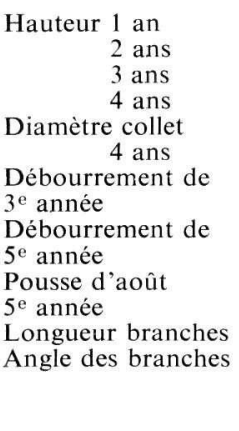 & $\begin{array}{l}\text { totalité } \\
\ll \\
\ll \\
\ll \\
\text { totalité } \\
\text { totalité } \\
\ll \\
\ll \\
\ll \\
\text { totalité } \\
\text { bloc } 1 \\
\text { bloc } 1\end{array}$ & $\begin{array}{l}\text { juin } 1968 \\
\text { octobre } 1968 \\
\text { mars } 1970 \\
\text { novembre } 1970 \\
\text { novembre } 1970 \\
\\
22.04 .69 \\
2.05 .69 \\
28.04 .71 \\
3.05 .71 \\
\text { août } 1971 \\
\text { avril } 1971 \\
\text { avril } 1971\end{array}$ & $\begin{array}{l}\text { au cm près } \\
\quad " \\
\text { "« } \\
\text { au mm près } \\
\text { pour la provenance MA-PA plus précoce } \\
\text { pour les provenances SJ-AV et GR-FA } \\
\text { pour MA-PA seulement } \\
\text { pour SJ-AV et GR-FA seulement } \\
\text { présence ou absence sont notées } \\
\text { longueur des branches du dernier verticille (4 ans) } \\
\text { angle d'insertion d'une branche de ce verticille } \\
\text { mesuré à l'aide d'un parallélogramme articulé } \\
\text { supportant un rapporteur. }\end{array}$ \\
\hline
\end{tabular}

Le Douglas n'a pas une organisation aussi nette que le Sapin et la distinction des branches appartenant à un verticille est parfois ambiguë surtout à la partie inférieure du verticille. Afin de lever cette ambiguité, on a fixé arbitrairement à $2 \mathrm{~cm}$ la longueur d'insertion sur la tige des branches d'un verticilie, les branches présentant un nıveau d'insertion inférieur, n'étant pas prises en considération.

Le terme de l'essai a été fixé à l'issue de la $6^{\mathrm{C}}$ année : des observations supplémentaires seront alors réalisées, notamment les poids secs de certains organes : tige, système racinaire, etc..., ainsi que la densité du bois.

\section{5. - Méthodes d'analyse}

La presque totalité des données a été traitée par la Station de Biométrie du C.N.R.F. ${ }^{1}$, au moyen des programmes suivants :

- Histogramme des mesures,

- Moyennes et paramètres de dispersion $)$
Corrélations entre caractères
, par parcelle unitaire

- Analyse de variance à 3 facteurs.

1. Les calculs ont été réalisés sous la responsabilité de M. Millier que nous tenons à remercier ici. 


\section{II. - RÉSUlTATS ET DISCUSSION}

Il convient de noter que la durée de l'expérience excède le temps de séjour du Douglas en pépinière; les plants de Douglas sont habituellement produits en 2 ans de semis, 1 an de repiquage ou 1 an de semis, 2 ans de repiquage. Cette longue durée n'a été choisie que pour les besoins de l'expérience.

\section{1. - Effet de la densité sur la mortalité}

Dans toutes les figures où la densité est en abscisse, la densité (ou l'écartement) exprimée est la densité réelle qui tient compte des mortalités intervenues et non la densité réelle au semis.

En effet, aux fortes densités, une mortalité importante est apparue notamment en $4^{\mathrm{e}}$ année, où la compétition entre les individus devient très intense. Pour la provenance Manning Park, la mortalité est moins élevée que pour les deux autres Granite Falls et StJust d'Avray (fig. 1). En effet, ces deux dernières sont plus vigoureuses et la compétition entre individus y est plus vive.

\section{2. - Effet de la densité sur la vigueur}

La vigueur est appréciée par les caractères suivants : hauteur totale moyenne et pousses annuelles moyennes, diamètre moyen au collet. L'effet de la compétition peut se traduire sur la grandeur des caractères, mais également sur la répartition et la variabilité de ces caractères. Nous envisagerons successivement ces deux points.

\subsection{La grandeur des caractères}

\subsection{Hauteur totale de la tige à $1,2,3,4$ ans.}

La figure 1 donne la hauteur de la tige à 1, 2, 3, 4 ans. On peut remarquer que l'effet dépressif de la densité se fait sentir dès la deuxième année et s'accroît par la suite, ceci étant moins sensible pour la provenance Manning Park, moins vigoureuse.

L'analyse de la variance réalisée suivant le schéma ci-après conduit au test $F$ résumé dans le tableau 3.

\begin{tabular}{l|c} 
Origine de la variation & Degrés de liberté \\
\hline & \\
Provenance P $\ldots \ldots \ldots \ldots$ & 2 \\
Espacement E $\ldots \ldots \ldots \ldots$ & 2 \\
Interaction P $\times$ E & 4 \\
Bloc $\ldots \ldots \ldots \ldots$ & 3 \\
Résiduelle $\ldots \ldots \ldots \ldots \ldots$ & 24 \\
\hline
\end{tabular}



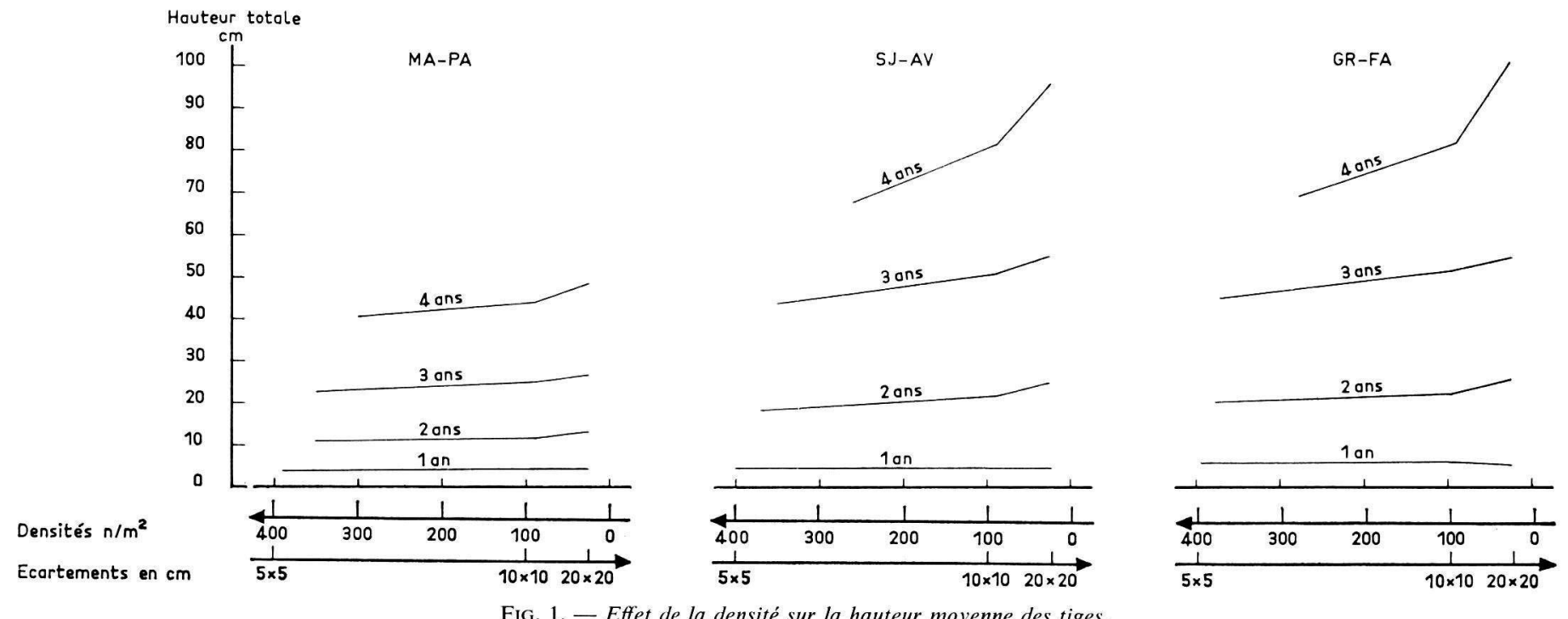

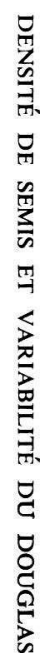

Fig. 1. - Density effect on the mean stem height 
L'effet provenance apparaît significatif dès la première année : ceci ne saurait nous surprendre, compte tenu des résultats acquis antérieurement dans les plantations comparatives où ces trois provenances sont représentées. L'effet de la densité se fait sentir dès la deuxième année : les deux provenances Granite Falls et St-Just d'Avray semblent avoir un comportement identique vis-à-vis de la densité.

TABLEAU 3 - TABLE 3

Degrés de signification de l'effet provenance, espacement, interaction et bloc sur les différents caractères.

Significance degrees of the provenance, spacing, interaction provenance $x$ spacing, and block for the various characters.

\begin{tabular}{|c|c|c|c|c|}
\hline & $\begin{array}{c}\text { Provenances } \\
\text { P }\end{array}$ & $\begin{array}{c}\text { Espacement } \\
\text { E }\end{array}$ & $\begin{array}{l}\text { Intreaction } \\
\qquad \mathrm{P} \times \mathrm{E}\end{array}$ & Bloc \\
\hline 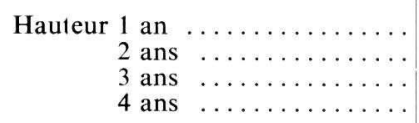 & $\begin{array}{l}\mathrm{XX} \\
\mathrm{XX} \\
\mathrm{XX} \\
\mathrm{XX}\end{array}$ & $\begin{array}{l}\mathrm{NS} \\
\mathrm{XX} \\
\mathrm{XX} \\
\mathrm{XX}\end{array}$ & $\begin{array}{l}\text { NS } \\
\text { NS } \\
\text { NS } \\
\text { XX }\end{array}$ & $\begin{array}{l}\mathrm{XX} \\
\mathrm{XX} \\
\mathrm{XX} \\
\mathrm{XX}\end{array}$ \\
\hline 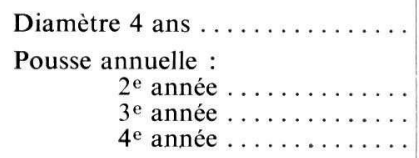 & $\begin{array}{l}X X \\
X X \\
X X\end{array}$ & $\begin{array}{l}x x \\
x x \\
x x \\
X X\end{array}$ & $\begin{array}{l}\text { NS } \\
\text { X } \\
\text { NS } \\
\text { XX }\end{array}$ & $\begin{array}{l}\mathrm{XX} \\
\mathrm{XX} \\
\mathrm{XX} \\
\mathrm{NS}\end{array}$ \\
\hline
\end{tabular}

XX Différences significatives au seuil de 1 p. cent

$X$ Différences significatives au seuil de $5 \mathrm{p}$. cent

NS Pas de différences significatives.

On peut en effet remarquer un effet bloc constant et surtout une interaction provenance $\times$ espacement significative à 4 ans; ceci tendrait à prouver que le comportement des provenances vis-à-vis de la densité est différent. En fait il n'en est rien, cette interaction apparente étant due à la faible croissance de Manning Park. En effet, si l'on compare les provenances à hauteur égale, hauteur à 3 ans pour St-Just d'Avray et Granite Falls, hauteur à 4 ans pour Manning Park (fig. 1), le comportement des 3 provenances apparaît identique.

Toutefois, la variable hauteur totale est critiquable dans la mesure où elle est cumulative, c'est-à-dire qu'elle intègre les pousses de plusieurs années. Le choix de la variable pousse annuelle est peut-être plus précis pour déceler l'apparition de l'effet de la densité.

\subsection{Pousse annuelle, $2^{\mathrm{e}}, 3^{\mathrm{e}}, 4^{\mathrm{e}}$ année.}

L'examen du caractère pousse annuelle moyenne (cf. fig. 2) conduit aux mêmes conclusions que le caractère hauteur : indépendamment de l'effet provenance, l'effet de la densité se traduit par une forte diminution de la longueur de la pousse. Les interactions prove- 


\section{Pousse onnuelle}
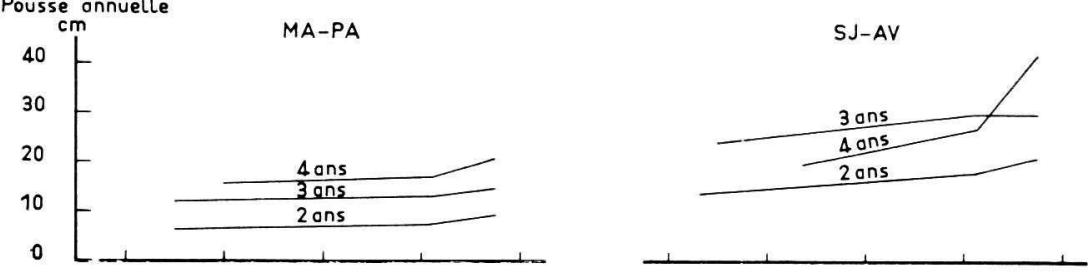

FIG. 2. - Effet de la densité sur la longueur des pousses annuelles.

FIG. 2.- Density effect on the mean-length_of the annual leader-shoot
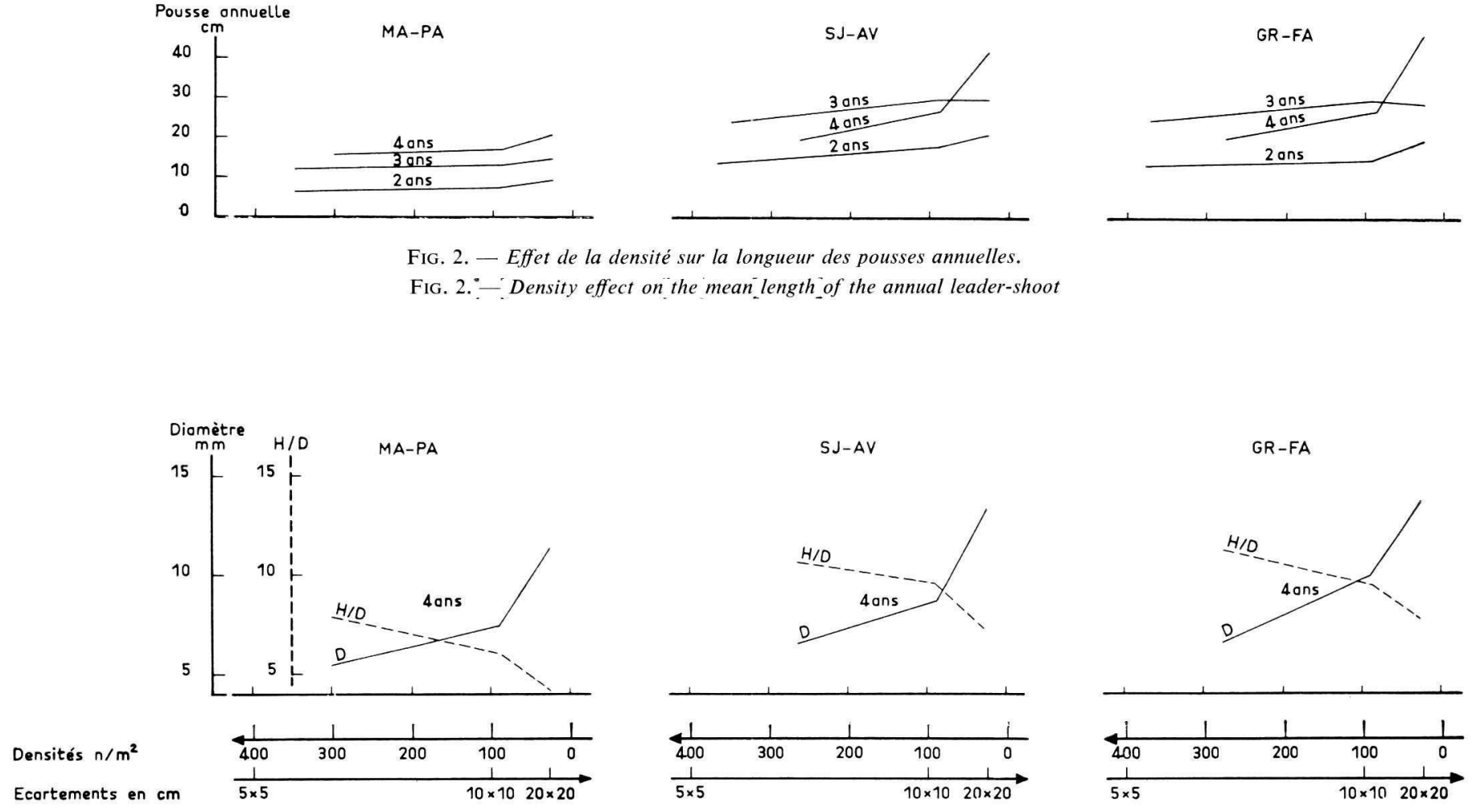

SJ-AV
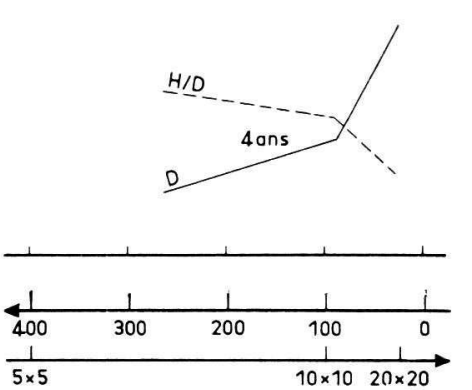

GR-FA

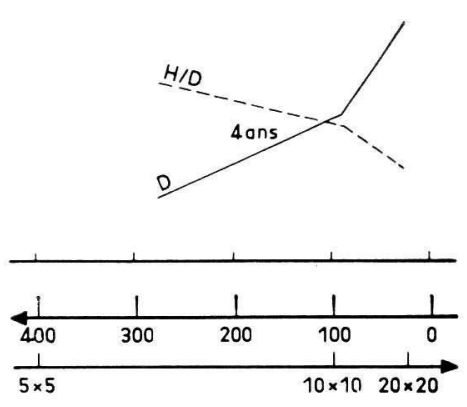

FIG. 3. - Effet de la densité sur le diamètre moyen au collet, et sur la forme de la tige appréciée par le rapport hauteur/diamètre.

FIG. 3. - Density effect on the mean collar diameter and the slenderness of the stem assessed by the ratio height/diameter 
nances $\times$ espacements, apparemment significatives, doivent être interprétées comme précédemment.

Pour la provenance Manning Park, la longueur de la pousse annuelle augmente chaque année, à une densité donnée.

Pour Granite Falls et St-Just d'Avray, cette évolution est identique à l'écartement $20 \times 20$. Par contre à l'écartement $5 \times 5$ et $10 \times 10$, la pousse de $4^{\mathrm{e}}$ année est très nettement inférieure à la pousse de $3^{\mathrm{e}}$ année. Ceci traduit l'effet de compétition entre individus probablement beaucoup plus intense en $4^{\mathrm{e}}$ année.

Pour une année donnée, la longueur de la pousse augmente avec l'écartement. Cependant pour Granite Falls et St-Just d'Avray, la pousse annuelle de $3^{\mathrm{e}}$ année (1969) présente une égale vigueur aux écartements $10 \times 10$ et $20 \times 20$; ce résultat semble paradoxal comparativement aux résultats des années précédentes et de l'année suivante. Il faut admettre qu'un facteur est intervenu pour limiter la croissance, notamment à l'espacement de $20 \times 20$. Quel est ce facteur? On peut avancer l'explication suivante :

En 1969, 3e année, on peut noter après un mois de juin très pluvieux une sécheresse estivale très marquée notamment en juillet et septembre. Les plants, espacés de $20 \times 20$, plus vigoureux des provenances St-Just d'Avray et Granite Falls auraient donc été proportionnellement plus pénalisés que les autres, notamment dans la croissance des pousses d'août. En effet les observations effectuées par LACAZE et Tomassone (1967) ont montré que la provenance Manning Park cessait de croître précocement (15 juillet) et que, par contre, les provenances du piémont ouest de la Chaîne des Cascades (État de Washington) avaient une période de végétation beaucoup plus longue et une aptitude plus forte à la production des pousses d'août. Celles-ci ne s'expriment d'ailleurs que dans un environnement favorable.

\subsection{Diamètre à 4 ans - relation hauteur-diamètre.}

Le diamètre est généralement le caractère le plus «sensible » à l'effet de la densité. Les mesures de diamètre n'ont pas été prises régulièrement, ce qui ne permet pas d'étudier l'apparition de l'effet dépressif de la densité sur ce caractère.

L'analyse effectuée à 4 ans (tableau 3, fig. 3) montre très nettement une diminution du diamètre avec l'augmentation de la densité. La provenance Manning Park atteint pour ce caractère un niveau de développement très voisin de celui des 2 autres. L'effet de la densité est plus marqué pour le diamètre que pour la hauteur.

En effet, si l'on prend comme témoin l'espacement $20 \times 20 \mathrm{~cm}$, le diamètre moyen des plants espacés de $5 \times 5$ est de $50 \mathrm{p}$. cent inférieur alors que la différence est de $30 \mathrm{p}$. cent pour la hauteur du même âge, pour les provenances Granite Falls et St-Just d'Avray.

Le comportement des provenances vis-à-vis de la densité apparaît encore une fois très semblable.

La forme de la tige peut être appréciée par le rapport hauteur/diamètre. La provenance Manning Park apparaît plus trapue que les deux autres (fig. 3). Pour les 3 provenances on 
constate une augmentation du rapport hauteur/diamètre avec la densité : à forte densité, les plants sont plus grêles parce que la croissance en diamètre est finalement plus affectée par la compétition que la croissance en hauteur. Ici encore le comportement des 3 provenances apparaît très voisin.

L'intensité de la liaison Hauteur-Diamètre peut être appréciée par le coefficient de corrélation entre ces deux caractères. Après transformation de cette variable en la variable $\mathrm{Z}=$ Arg thr, aucune différence significative ne peut être mise en évidence entre les différents écartements et les différentes provenances.

\subsection{Distribution et variabilité des caractères}

\subsection{Distribution des fréquences.}

Les calculs ont été réalisés sur l'ensemble des individus du même traitement pour les caractères :

Hauteur à 1, 2, 3, 4 ans;

Diamètre à 4 ans.

Pour chaque caractère, les individus ont été rangés en classes d'égale étendue, celle-ci étant variable suivant les caractères. L'examen de la fig. 4 permet de montrer que, indépendamment des moyennes :

a) Le diamètre au collet à 4 ans se distribue avec une dissymétrie à gauche au plus faible espacement. A $10 \times 10$ et $20 \times 20$, les courbes ont tendance à se normaliser. Le comportement des 3 provenances est sensiblement identique encore que Manning Park semble présenter la plus grande dissymétrie pour l'espacement $5 \times 5$ et également pour $10 \times 10$.

Koyama et Kira (1956), cités par Illy et Lemoine (1970), ont montré en particulier sur Pinus densiflora Thumb. planté à 4 espacements de 0,50 à $2,00 \mathrm{~m}$ que l'évolution vers la dissymétrie à gauche de la distribution de fréquences du poids de la plante, est d'autant plus rapide, que la densité de la population est importante. Ce phénomène se trouve accentué par la mortalité naturelle des plants; par contre, la distribution des hauteurs reste de type normal, assez longtemps.

Pour Delvaux (1966), la dissymétrie à gauche traduit une nutrition difficile et apparaît surtout pour le diamètre et peu pour les hauteurs.

Bien que dans notre cas, il s'agisse de semis et non d'une plantation, il est clair que la dissymétrie observée traduit bien une situation de compétition. Il est regrettable que les mesures de diamètres antérieures à 4 ans n'aient pu être faites, afin de vérifier à partir de quel âge se faisait sentir cette dissymétrie.

b) En ce qui concerne les hauteurs, cette observation est beaucoup moins évidente. La persistance de la normalité du caractère hauteur est à rapprocher de sa moindre sensibilité à l'effet de la densité. 

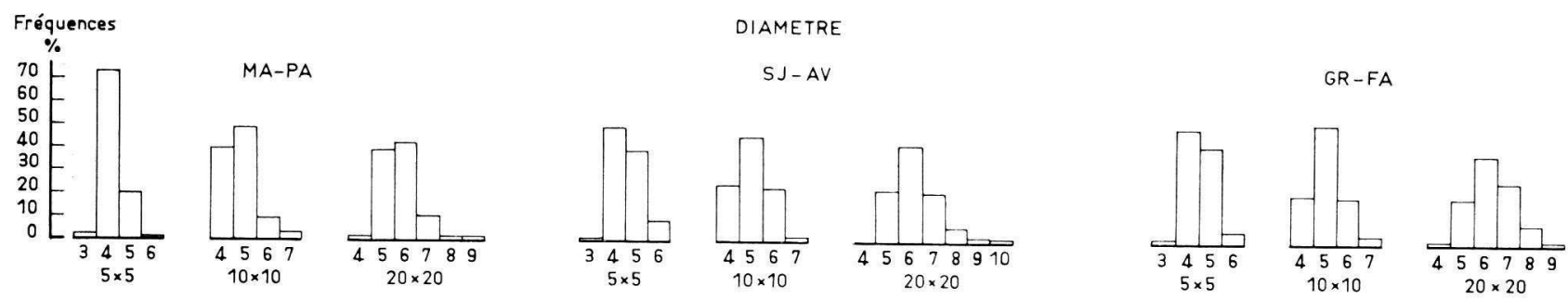

4 ans

Fréquences

HAUTEURS
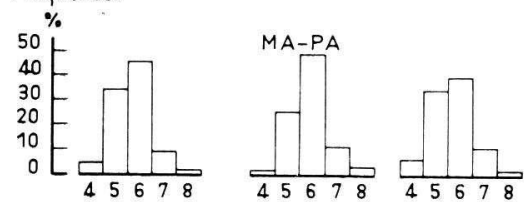

SJ-AV

GR-FA
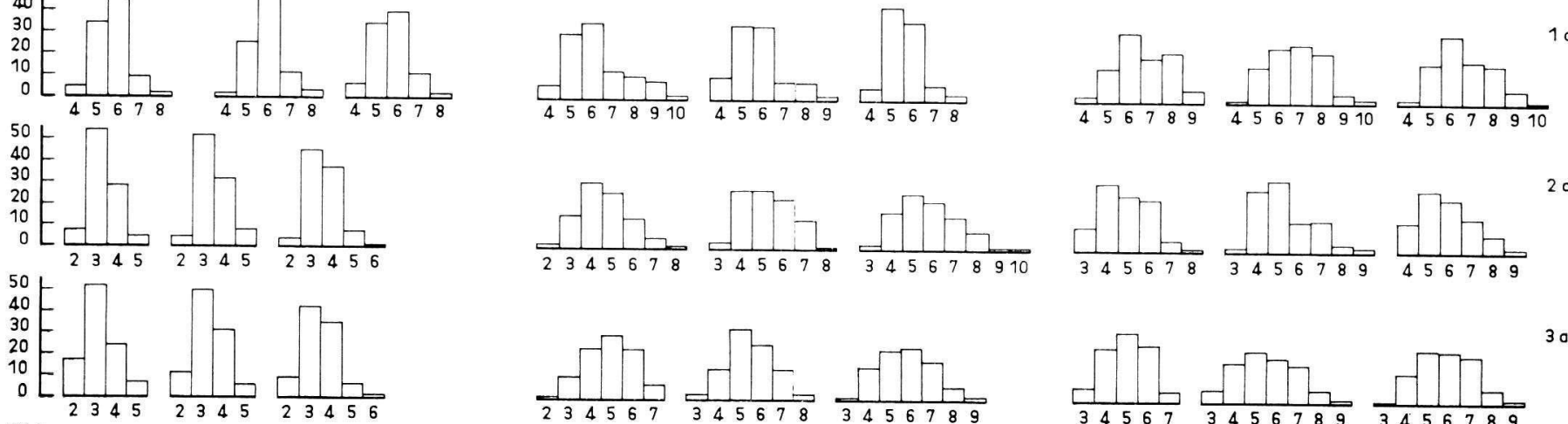

2 ans
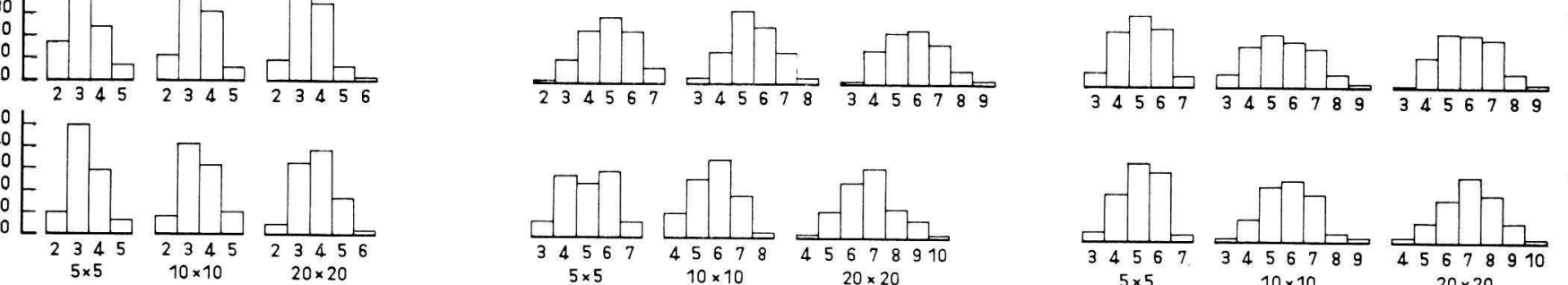

3 ons

FIG. 4. - Effet de la densité sur la distribution des fréquences du diamètre au collet et de la hauteur totale de la tige.

FIG. 4. - Density effect on the frequencies distribution of the collar diameter and the stem height 


\subsection{2. Écart-type et coefficients de variation.}

Il était intéressant de chercher si ces valeurs caractéristiques de la variabilité individuelle pouvaient exprimer la compétition et son apparition. Nous avons pour cela effectué les moyennes de ces valeurs à l'intérieur de chaque traitement.

a) Pour le diamètre à 4 ans (fig. 5), la diminution de la densité se traduit très clairement par une augmentation de l'écart-type. Il se trouve aussi que la valeur moyenne du diamètre varie dans le même sens. Il pourrait alors y avoir un certain effet d'échelle au sens de FALCONNER (1960). Cependant le coefficient de variation montre qu'il n'en est rien puisqu'il augmente avec la densité. L'essentiel de la variation se fait entre l'écartement $10 \times 10$ et l'écartement $20 \times 20$. La situation de compétition se trouve donc nettement exprimée par les deux variables écart-type, et coefficient de variation. Cependant les moyennes n'étant pas égales, on ne pourrait, en toute rigueur, comparer les écarts-types; quand au coefficien $t$ de variation, il ne peut fournir que des indications.

b) Pour la hauteur, (fig. 6), l'écart-type augmente lorsque la densité décroît, dès la $2^{\mathrm{e}}$ année, et ce phénomène s'intensifie les $3^{\mathrm{e}}$ et $4^{\mathrm{e}}$ années, surtout pour la provenance Granite Falls. Ici encore, compte tenu de la variation dans le même sens de la valeur moyenne de la hauteur, on peut penser à un éventuel effet d'échelle. Le calcul des coefficients de variation ne permet pas de confirmer ou d'infirmer cette hypothèse (fig. 7).

Dès la $2^{\mathrm{e}}$ année, on constate, une chute importante du coefficient de variation, fait très général après une période d'installation. Par contre, la relation coefficient de variation-densité n'est pas évidente.

Il convient de noter qu'aux fortes densités où des plants ont disparu, la répartition inégale des plants survivants sur le terrain peut perturber très probablement la valeur du coefficient de variation.

c) Si l'on choisit le caractère pousse annuelle, la fig. 8 montre que pour les trois provenances le coefficient de variation diminue de la $2^{\mathrm{e}}$ à la $3^{\mathrm{e}}$ année, ce qui traduit une " homogénéisation ». Par contre en $4^{\mathrm{e}}$ année, il remonte à des niveaux supérieurs ou égaux à ceux de la $3^{\mathrm{e}}$ année ou même de la $2^{\mathrm{e}}$ année, pour les plus fortes densités. Ceci est très net pour les provenances Granite Falls et surtout St-Just d'Avray, moins marqué pour Manning Parks, provenance moins vigoureuse. A l'écartement de $20 \times 20 \mathrm{~cm}$, le coefficient de variation diminue encore en $4^{\mathrm{e}}$ année.

d) Malgré l'inégalité des moyennes, on a tenté d'analyser les différences constatées entre les écarts-types après transformation logarithmique «normalisante ». La décomposition des variances utilisée est identique à celle énoncée précédemment. Les résultats sont donnés dans les tableaux 4 et 5 . 

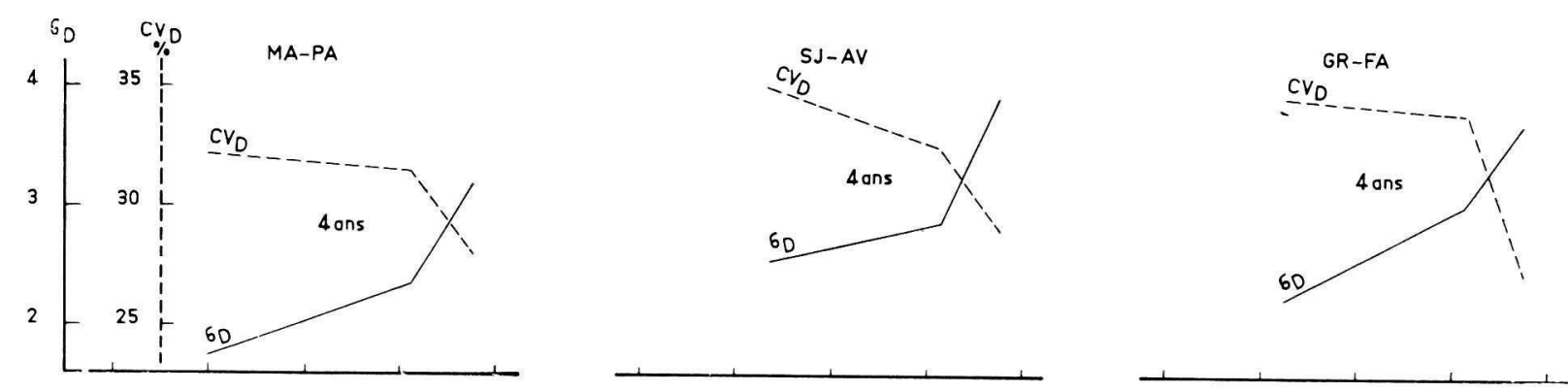

FIG. 5. - Effet de la densité sur l'écart type et le cafficient de variation du diametre au collet.

Fig. 5. - Density effect on the standard deviation and the variation cafficient of the collar diameter
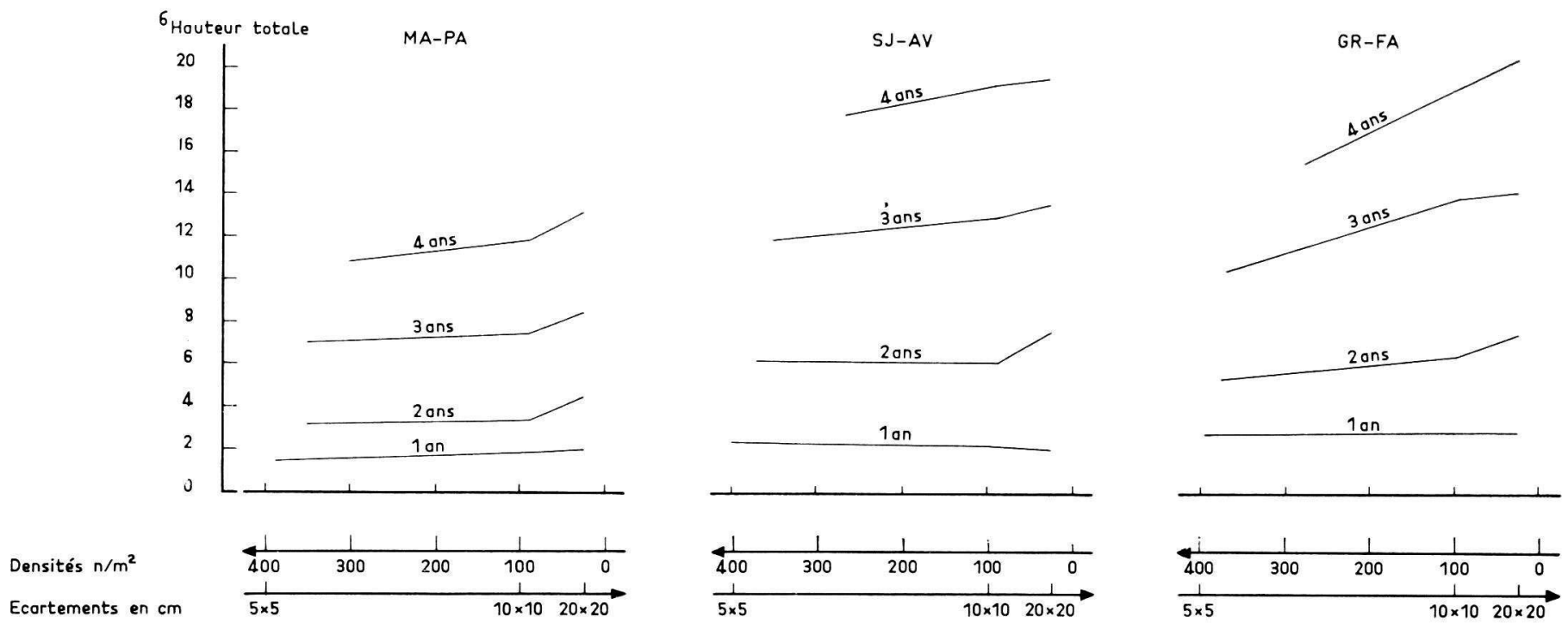

FIG. 6. - Effet de la densité sur l'écart type de la hauteur dèla tige.

FIG. 6. - Densitv effect on the standard deviation of the stem height 


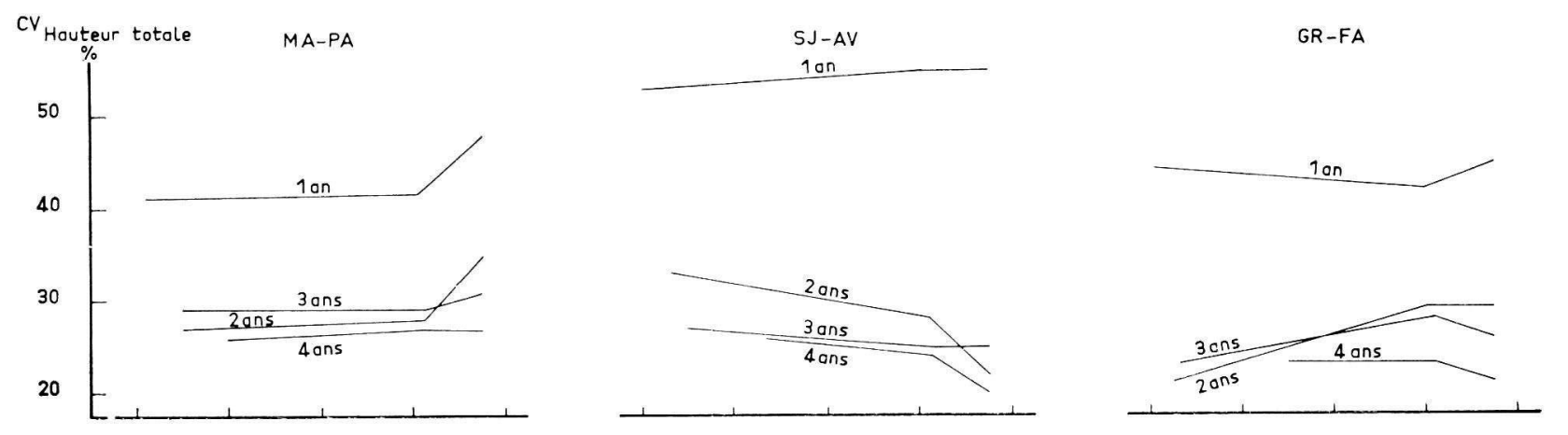

FIG. 7. - Effet de la densité sur le cefficient de variation de la hauteur de la tige.

FiG. 7. - Density effect on the variation cafficient of the stem height

Densités $\mathrm{n} / \mathrm{m}^{2}$

Coefficient de variation
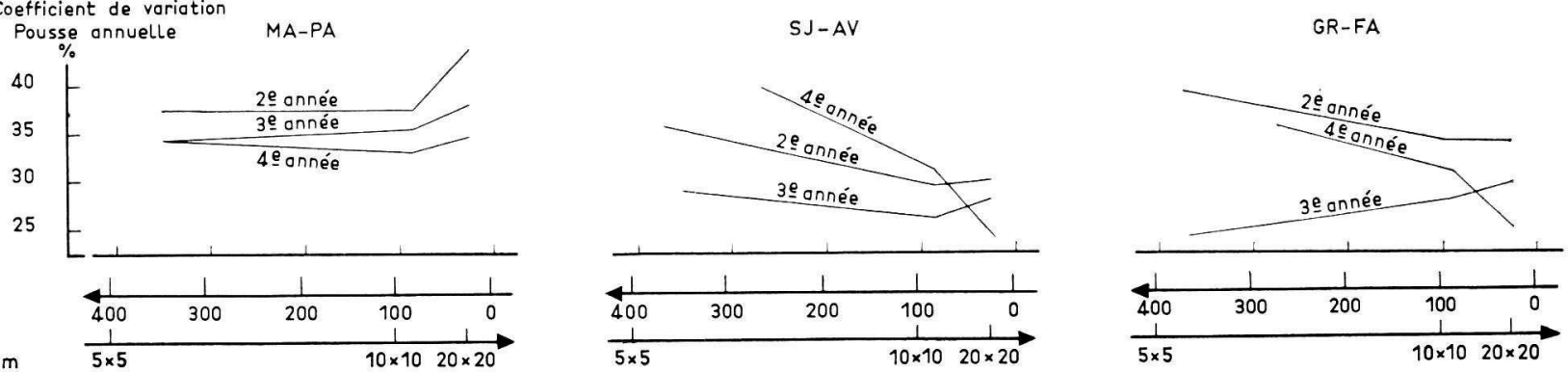

FIG. 8. - Effet de la densité sur le cafficient de variation de la longueur de la pousse annuelle.

FIG. 8. - Density effect on the variation cafficient of the lengh of the annual leadershoot 
TABLEAU 4 - TABLE 4

Logarithme de l'Écart-type des caractères mesurés Standard deviation logarithm for the various characters; F test

\begin{tabular}{|c|c|c|c|c|}
\hline Log. $\sigma$ & Provenance & Espacement & $\begin{array}{l}\text { Interaction } \\
\qquad P \times E\end{array}$ & Blocs \\
\hline 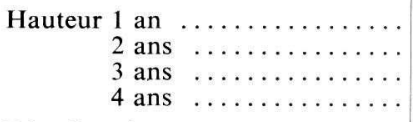 & $\begin{array}{l}X X \\
X X \\
X X \\
X X\end{array}$ & $\begin{array}{l}\mathrm{NS} \\
\mathrm{XX} \\
\mathrm{XX} \\
\mathrm{XX}\end{array}$ & $\begin{array}{l}\text { XX } \\
\text { NS } \\
\text { NS } \\
\text { NS }\end{array}$ & $\begin{array}{l}X X \\
X X \\
X \\
X\end{array}$ \\
\hline $\begin{array}{l}\text { Diamètre } 4 \text { ans } \ldots \ldots \ldots \ldots \ldots \\
\text { Pousse annuelle }: \\
2^{\mathrm{e}} \text { année } \ldots \ldots \ldots \ldots \ldots \ldots \\
3^{\mathrm{e}} \text { année } \ldots \ldots \ldots \ldots \ldots \ldots \ldots \\
4^{\mathrm{e}} \text { année } \ldots \ldots \ldots \ldots \ldots \ldots\end{array}$ & $\begin{array}{l}X X \\
X X \\
X X \\
X X\end{array}$ & $\begin{array}{l}X X \\
X X \\
X X \\
X X\end{array}$ & $\begin{array}{l}\text { NS } \\
\text { NS } \\
\text { NS } \\
\text { NS }\end{array}$ & $\begin{array}{l}\mathrm{XX} \\
\mathrm{NS} \\
\mathrm{X} \\
\mathrm{NS}\end{array}$ \\
\hline
\end{tabular}

Test F. - XX différence significative au seuil de 1 p. cent. $X$ différence significative au seuil de $5 \mathrm{p}$. cent. NS pas de différences significatives.

TABLEAU 5 - TABLE 5

Logarithme de l'Écart-type des caractères mesurés Standard deviation logarithm for the various characters; Duncan's test

\begin{tabular}{|c|c|c|c|c|c|c|c|c|}
\hline & $\begin{array}{c}\text { Log. } \sigma \\
\text { hauteur } \\
1 \text { an }\end{array}$ & $\begin{array}{l}\text { Log. }= \\
\text { hauteur } \\
2 \text { ans }\end{array}$ & $\begin{array}{c}\text { Log. }= \\
\text { hauteur } \\
3 \text { ans }\end{array}$ & $\begin{array}{c}\text { Log. } \sigma \\
\text { hauteur } \\
4 \text { ans }\end{array}$ & $\begin{array}{c}\text { Log. } \sigma \\
\text { diamètre } \\
4 \text { ans }\end{array}$ & $\begin{array}{c}\text { Log. } \\
\text { pousse } \\
2^{\mathrm{e}} \text { année }\end{array}$ & $\begin{array}{c}\text { Log. } \sigma \\
\text { pousse } \\
3^{\mathrm{e}} \text { année }\end{array}$ & $\begin{array}{c}\text { Log. } \sigma \\
\text { pousse } \\
4^{\mathrm{e}} \text { année }\end{array}$ \\
\hline $\begin{array}{r}20 \times 20 \\
10 \times 10 \\
5 \times 5\end{array}$ & & & & & & & & \\
\hline
\end{tabular}

Test de Duncan (seuil 1 p. cent) (toutes provenances confondues) Le trait vertical indique l'absence de différences significatives.

Pour l'écart-type des hauteurs, l'effet provenance est sensible dès la première année. L'effet espacement apparaît en $2^{\mathrm{e}}$ année où paradoxalement c'est entre les écartements $10 \times 10$ et $20 \times 20$ que la différence est significative. En $3^{\mathrm{e}}$ année et $4^{\mathrm{e}}$ année, la seule différence significative est entre les deux écartements extrêmes $5 \times 5$ et $20 \times 20$. L'interaction significative provenance $\times$ espacement trouvée en première année n'est pas interprétable, les semis étant très jeunes et la compétition certainement très peu marquée.

L'écart-type des pousses annuelles accuse des différences significatives dès la $2^{\mathbf{e}}$ année, mais les espacements confondus sont tantôt $5 \times 5,10 \times 10$ tantôt $10 \times 10,20 \times 20$. L'effet provenance est constant. 
Pour l'écart-type du diamètre, les différences sont très fortes. Ceci ne fait que confirmer l'action prépondérante de la compétition sur le diamètre.

L'effet provenance existe comme pour les autres caractères.

L'écart-type et surtout le coefficient de variation peuvent donc constituer des " indicateurs » valables de l'existence d'une situation de compétition.

\subsection{Conclusions}

L'effet de la densité sur la vigueur se manifeste très précocement : dès la $2^{\mathrm{e}}$ année pour la croissance en hauteur. Pour le diamètre, on peut supposer que cet effet est encore plus précoce.

Contrairement à l'opinion généralement répandue chez les praticiens, l'état serré ne fait pas « filer » le plant en hauteur. Tout en diminuant le diamètre, il diminue aussi la hauteur, dans des proportions moindres toutefois.

A travers les trois provenances étudiées, le Douglas se présente donc comme une espèce très sensible à l'effet de la densité. On rappellera qu'il est très sensible aux variations de la fertilité : c'est une espèce relativement exigeante sur le plan de la nutrition. Or il s'agit bien, au moins dans les premiers temps, d'une compétition au niveau racinaire. D'après les travaux de Eversole (1955), cité par Bartoli et Decourt (1971), il semble que chez le Douglas la densité de plantation ait un effet sensible sur la hauteur. BARToli et DeCOURT font d'ailleurs l'hypothèse que le Douglas serait une espèce particulièrement sensible à l'effet de la densité alors que l'Epicéa le serait beaucoup moins.

Indépendamment de la valeur moyenne des caractères, la densité influe également sur leur distribution : écart-type et coefficients de variation se trouvent modifiés.

Cependant, il n'est pas possible de mettre en évidence des différences dans le comportement vis-à-vis de la densité des trois provenances étudiées pour les caractères de vigueur. L'échantillonnage des provenances étant limité, on ne peut conclure toutefois à l'absence de variabilité intraspécifique chez le Douglas soumis à un effet de densité.

Sur un plan général, certains enseignements pratiques peuvent être tirés :

- l'utilisation de fortes densités est une technique parfois recommandée par certains pépiniéristes. Ceci conduit à diminuer la taille des plants et à en augmenter l'hétérogénéité au moins pour le caractère diamètre, le coefficient de variation augmentant lorsque la densité croît. Le pourcentage de plants rangés dans les meilleures classes des normes de qualité se trouve réduit. En outre aux fortes densités les plants sont plus grêles (rapport H/D fort), et beaucoup d'entre eux se trouvent éliminés des normes.

- pour les dispositifs expérimentaux de pépinière, ces résultats nous incitent à adopter des écartements plus grands que ceux utilisés jusqu'à présent $(12,5 \times 12,5 \mathrm{~cm})$. En effet nous constatons ici des différences de vigueur entre les écartements $10 \times 10$ et $20 \times 20$ qui sont significatives dès la $2^{\mathrm{e}}$ année.

Nous devons donc adopter au repiquage des espacements assez grands sous peine de voir s'introduire dans nos comparaisons un certain nombre de biais dus à ce que :

- les performances des provenances se trouvent en partie conditionnées par l'action de la densité, 
- les variances sont également modifiées.

Ce sont évidemment les provenances les plus vigoureuses qui sont les plus handicapées et l'expression de leur qualité génétique est ainsi sous-estimźe.

\section{3. - Étude de la compétition au niveau individuel}

DONAld (1963) cité par Illy et LemOINE (1970) définit ainsi la concurrence : « il y a concurrence entre deux ou plusieurs organismes lorsque chacun d'eux cherche à se procurer le montant nécessaire à ses besoins d'un facteur ou d'une chose qui sont fournis immédiatement en quantité inférieure aux exigences combinées de ces organismes ". Il y a donc un effet négatif sur l'un des deux (ou sur les deux) en situation de compétition.

On a vu que l'action de la densité se manifeste très précocement sur la hauteur. Pour le caractère diamètre, la dissymétrie à gauche des diagrammes de répartition des fréquences signifie qu'en situation de forte compétition il y a moins d'individus dominants que d'individus dominés.

A quel moment apparaît cet effet négatif d'un individu sur l'autre? On peut apporter un élément de réponse en étudiant la stabilité des classements phénotypiques individuels.

Pour les caractères hauteurs et pousses annuelles on peut utiliser comme critère les coefficients de corrélations au niveau individuel entre les perfomances de $1^{\text {re }}$ et de $4 \mathrm{e}$ année. Pour toutes les provenances ces coefficients sont significatifs mais faibles $\left(0,30<\mathrm{r}_{\mathrm{H}_{1}}, \mathrm{H}_{4}<\right.$ $0,54)$; on ne note pas de différences significatives entre espacements, ni entre provenances. Les coefficients de corrélation Hauteur à 2 ans, Hauteur à 4 ans, toujours au niveau individuel sont significatifs mais nettement plus élevés (de 0,61 à 0,77 ), les trois provenances ayant un comportement identique.

Cependant, la sécheresse de 1969 ( $3^{\mathrm{e}}$ année) a apporté certainement des biais dans la croissance : les coefficients de corrélations entre pousses de $2^{\mathrm{e}}$ et de $3^{\mathrm{e}}$ années d'une part, de $3^{e}$ et de $4^{\mathrm{e}}$ années d'autre part ne sont pas très élevés bien que significatifs (de 0,23 à 0,56). Pour les provenances Granite Falls et St-Just d'Avray le coefficient de corrélation entre les pousses de $3^{\mathrm{e}}$ et $4^{\mathrm{e}}$ année diminue nettement aux espacements plus grands. A ces espacements Manning Park a un coefficient de corrélation plus élevé que les deux autres provenances, ce qui confirme les hypothèses faites à propos de la sécheresse 1969.

Pour les caractères de croissance en hauteur les performances de $4^{\mathrm{e}}$ année semblent donc déterminées dès la $1^{\text {re }}$ année et surtout la $2^{\mathrm{e}}$ année. Mais on n'observe aucune variation bien nette en fonction de la densité.

Tout se passe finalement comme si le classement initial, basé sur des facteurs génétiques ou environnementaux (les effets de compétition était peu marqués dans ceux-ci), se trouvait ensuite « fixé » par la compétition.

\section{4. - Effet de la densité sur la ramification}

Les mesures effectuées pour un seul bloc sur le verticille de 4 ans ne permettent pas de conclusions claires quant à l'angle d'insertion des branches. Par contre la longueur des 
branches de ce verticille est fortement affectée par la densité (cf. fig. 9). Pour les provenances Granite Falls et St-Just d'Avray elle peut varier du simple au double de la plus forte à la plus faible densité. Pour Manning Park, provenance moins vigoureuse, les différences sont moins marquées.

On peut rattacher la croissance annuelle de la flèche à celle des branches. Le tableau 6 donne pour la $4^{\mathrm{e}}$ année les coefficients de corrélation au niveau individuel entre ces deux caractères.

TABLEAU 6 - TABLE 6

Coefficients de corrélation entre la croissance de la flèche et les branches de $4^{\mathrm{e}}$ année (dernier verticille)

Coefficients of correlation between the leader-shoot growth and the branchs growth at the 4th year (last whorl)

\begin{tabular}{|c|c|c|c|}
\hline \multirow{2}{*}{ Écartement } & \multicolumn{3}{|c|}{ Provenances } \\
\hline & MA-PA & SJ-AV & GR-FA \\
\hline $5 \times 5$ & 0,699 & 0,550 & 0,584 \\
\hline $10 \times 10$ & 0,691 & 0,647 & 0,610 \\
\hline $20 \times 20$ & 0,682 & 0,702 & 0,366 \\
\hline Ensemble & 0,807 & 0,862 & 0,796 \\
\hline
\end{tabular}

Tous ces coefficients sont significatifs : le développement des branches semble dont lié à la vigueur générale du plant. Dans l'ensemble, il y a peu de différences entre provenances ou espacements. Cependant, pour la provenance Granite Falls, le coefficient de corrélation pousse terminale-pousse latérale est faible à l'espacement de $20 \times 20 \mathrm{~cm}$.

Le calcul des droites de régression entre les deux caractères a été fait sur chaque parcelle unitaire du bloc observé. On obtient un faisceau de droites très proches les unes des autres. Leurs pentes sont très voisines : les droites représentatives des parcelles à faible densité se trouvent à la partie supérieure du faisceau. Au niveau provenance (les espacements étant confondus), les trois droites sont très proches. A ce stade encore juvénile du développement, il semble donc exister une loi de morphogénèse inscrite dans l'espèce.

La croissance des branches et de la flèche est liée alors au niveau de nutrition du plant lui-même affecté par la densité dont les effets peuvent être :

- une compétition racinaire pour l'eau et les éléments minéraux,

- compétition aérienne pour la lumière qui peut entraîner des réductions de photosynthèse et des perturbations physiologiques (synthèse des auxines). 


\section{5. - Effet de la densité sur certains caractères phénologiques}

\subsection{Le débourrement végétatif.}

Il a été observé en $3^{\text {e }}$ et $5^{\mathrm{e}}$ années. Le caractère retenu est le débourrement du bourgeon terminal, noté débourré ou non. Compte tenu des grandes différences de précocité entre provenances (Manning Park est beaucoup plus précoce), les observations ont été faites à des dates différentes. Les niveaux de débourrement ne sont pas comparables entre Manning Park et les deux autres provenances; bien évidemment ils ne sont pas comparables d'une année sur l'autre. Les résultats sont donnés dans la fig. 10.

Pour les provenances Granite Falls et St-Just d'Avray, on note en $3^{\mathrm{e}}$ année une diminution importante de la précocité, en particulier entre les espacements $10 \times 10$ et $20 \times 20$. En $5^{\mathrm{e}}$ année, c'est l'inverse : les plants à grands écartements sont les plus précoces. Comment expliquer ces résultats apparemment contradictoires?

LACAZE (1969) a montré pour l'Epicéa qu'il existait une liaison positive entre tardiveté et hauteur au niveau «moyenne de provenance ». En se plaçant au niveau individuel; il constatait que le coefficient de corrélation entre vigueur et tardiveté du débourrement est variable suivant les provenances, positif dans certains cas, faible ou nul dans d'autres.

LaCAzE et Arbez (1971), travaillant sur des descendances d'Epicéa issues de plusieurs provenances, ont pu montrer que les corrélations dues à l'environnement (qui comprennent outre des effets dus au milieu, des effets génétiques non additifs), expriment une liaison toujours négative entre précocité et vigueur. « Le bourgeon terminal, chez l'Épicéa, débourrerait d'autant plus tardivement que sa distance au sol est plus grande, donc que l'arbre est plus grand, même si l'écart de hauteur est seulement dû à l'action du milieu ».

Utilisant cette idée, nous avons étudié le débourrement au niveau individuel, en considérant pour les deux années concernées, les 10 plus grands et les 10 plus petits plants de chaque parcelle unitaire. Les résultats sont exprimés par les fig. 11 et 12. On peut les résumer ainsi :

a) explication des différences de débourrement entre la $3^{\mathrm{e}}$ et la $5^{\mathrm{e}}$ année :

En $3^{\mathrm{e}}$ année, on constate pour les trois provenances que les petits plants sont nettement plus précoces que les grands; ceci confirme les résultats obtenus pour l'Épicéa. Par contre, en $5^{\mathrm{e}}$ année, dans les provenances St-Just d'Avray et Granite Falls, les petits plants sont plus tardifs que les grands aux écartements $5 \times 5$ et $10 \times 10$, plus précoces à l'écartement $20 \times 20$. Dans la situation où la compétition est la moins forte, c'est donc «l'effet hauteur » du bourgeon terminal qui déterminerait en partie le débourrement comme en $3^{\mathrm{e}}$ année. En $5^{\mathrm{e}}$ année, la compétition est devenue plus forte aux écartements $5 \times 5$ et $10 \times 10$. Ce serait donc le niveau général de nutrition du plant qui dominerait. L'effet de compétition apparaît ainsi plus prononcé chez Granite Falls que chez St-Just d'Avray. Pour la provenance Manning Park, l'observation du débourrement en $5^{\mathrm{e}}$ année a été un peu tardive; dans une situation moins forte de concurrence, ce serait tout de même l'effet hauteur qui jouerait.

La faiblesse de la nutrition peut expliquer, aux fortes densités, la tardiveté des petits plants en $5^{\mathrm{e}}$ année. En effet,

- les petits plants sont largement dominés; en plus de la compétition racinaire, le manque de lumière diminue la photosynthèse, perturbe la formation des auxines et leur action; 


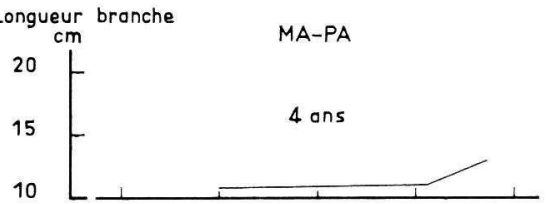

Fig. 9. - Effet de la densité sur la longueur des branches.

FIG. 9. - Density effect on the lenght of the branchs

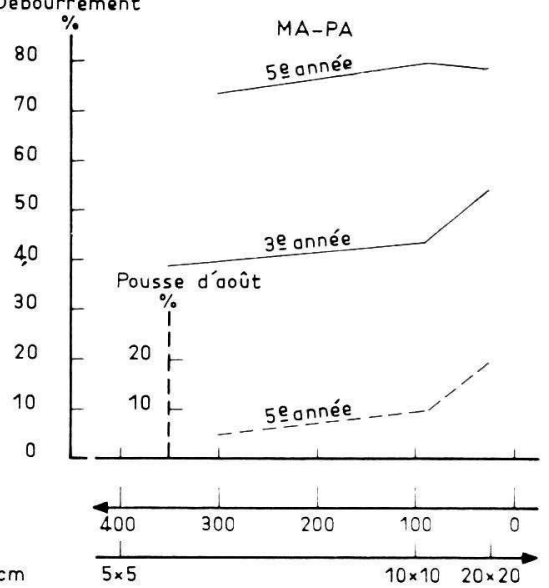

\section{SJ-AV}

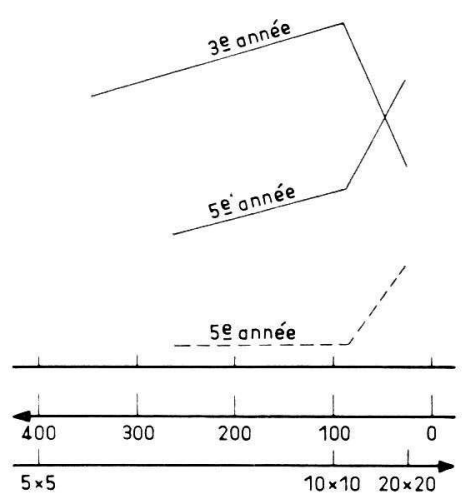

GR-FA

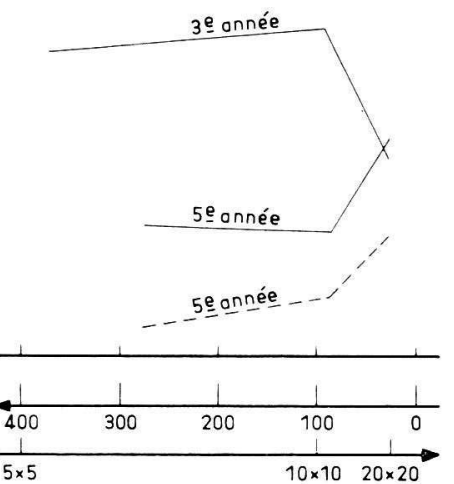

Ecartements en cm

FIG. 10. - Effet de la densité sur le débourrement et la production des pousses d'août. FIG. 10. - Density effect on the flushing and the lamma shoots production (3th and 5th year) 

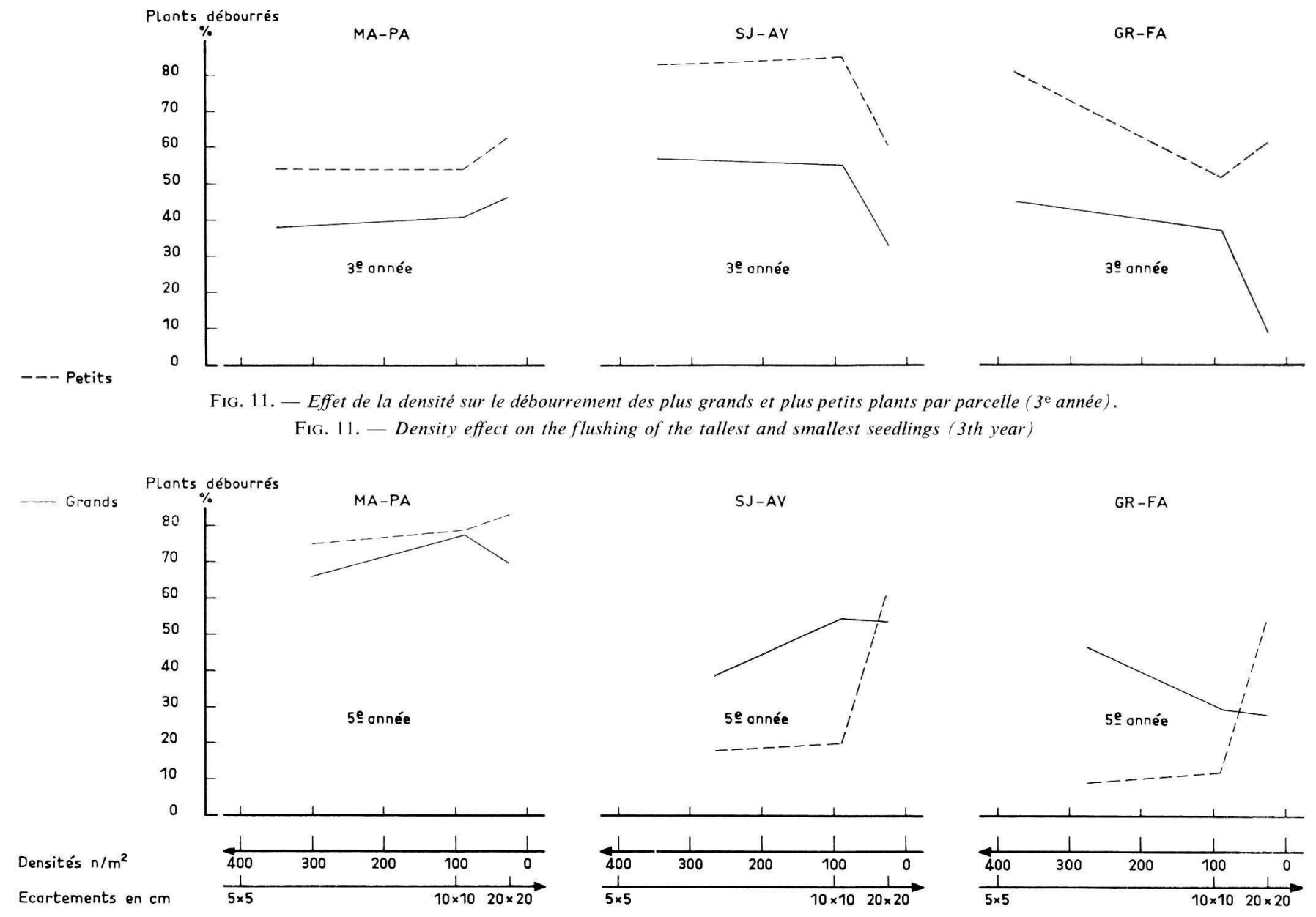

Fig. 12. - Effet de la densité sur le débourrement des plus grands et plus petits plants par parcelle ( $5 \mathrm{e}$ année). 
- à l'inverse, le reboiseur constate fréquemment une augmentation de la précocité du débourrement avec la fertilisation.

L'action de la température est difficile à retenir compte tenu de la très faible dimension des parcelles.

b) Variations constatées à l'intérieur des provenances suivant la densité :

- en $3^{\mathrm{e}}$ année :

Il n'y a pas de différences significatives entre les densités pour la provenance Manning Park. Pour la provenance St-Just d'Avray, la tardiveté augmente fortement entre l'écartement $10 \times 10$ et $20 \times 20 \mathrm{~cm}$, pour les petits et grands plants. C'est donc «l'effet hauteur » qui joue, puisque la taille moyenne des petits et grands plants augmente avec l'écartement. La conséquence pratique évidente est que le pépiniériste et le reboiseur doivent s'efforcer, en jouant sur la densité et la fertilisation notamment, d'obtenir les plants les plus grands possible pour échapper aux gelées tardives.

Pour Granite Falls, il en est de même pour les grands plants; les petits plants semblent réagir différemment toutefois.

- en $5^{\mathrm{e}}$ année :

Pour Manning Park, il n'y a pas de différences significatives du débourrement suivant la densité. L'observation du débourrement était cependant trop tardive.

Pour Granite Falls et St-Just d'Avray, la tardiveté du débourrement des petits plants augmente très fortement entre les espacements de $10 \times 10$ et $20 \times 20$. Pour les grands plants, l'amplitude de la variation du débourrement avec la densité est faible : les différences ne sont d'ailleurs pas significatives.

En conclusion, le débourrement du bourgeon terminal semble conditionné à la fois par la hauteur du plant et par la fertilité au sens large. Les biais apportés par la densité sont considérables.

Ces résultats ont une importance très grande pour les tests en pépinière, qu'ils soient comparatifs de provenances ou de descendances. Ici encore, il faut adopter au repiquage un écartement suffisant pour éviter tout biais dans les classements; l'écartement de $12,5 \times 12,5$ fréquemment utilisé est trop faible.

Pour les grandes espèces françaises de reboisement, Épicéa et Douglas, l'un des principaux critères de sélection individuelle est la tardiveté du débourrement végétatif. Cette sélection est logiquement contrôlée par les tests de descendances préalables à la réalisation des vergers à graines. Il faut être sûr qu'à ce niveau aucun biais ne modifie l'expression du génotype. Les résultats présentés peuvent nous y aider.

\subsection{Formation de pousses d'août.}

L'aptitude à la formation de pousses d'août a été observée en $5^{\mathrm{e}}$ année. Les résultats exprimés par la fig. 10 montrent que pour les trois provenances, le taux d'individus présentant des pousses d'août augmente avec l'écartement, donc avec la vigueur des plants; ce résultat est très classique, (cf. Walters J., Soos J., 1961). En outre, le niveau de fertilité conditionne également l'apparition des pousses d'août (Walters J., KozaK A., 1967). Ces deux raisons expliquent donc pour les trois provenances les résultats observés; il n'y a pas de différence de comportement entre les trois provenances étudiées. 


\section{CONCLUSIONS}

Les résultats de cette expérience montrent qu'il n'est pas possible de mettre en évidence des différences importantes dans le comportement vis-à-vis de la compétition des trois provenances étudiées. Mais l'échantillonnage de provenances est trop limité pour généraliser cette conclusion à l'espèce Douglas. En effet, sur les trois provenances, deux ont un comportement très voisin, St-Just d'Avray et Granite Falls, à tel point même que l'on peut se demander si St-Just d'Avray ne serait pas originaire de la même région de provenances, le piémond ouest de la Chaîne des Cascades. La comparaison ne peut donc plus se faire que sur la base de deux provenances : Manning Park et Granite Falls par exemple.

Cependant un certain nombre de points importants ont été établis :

a) La densité fait sentir très tôt son effet dépressif non seulement sur le diamètre des plants, ce qui est classique, mais encore sur leur hauteur, résultat moins souvent mis en évidence.

b) Le débourrement végétatif est fortement influencé par la densité, par l'intermédiaire de deux facteurs, la hauteur des plants et leur niveau de nutrition.

Sur le plan pratique, il faut retenir que :

- le pépiniériste devrait utiliser des densités de semis et surtout de repiquage qui laissent les plants hors concurrence. Le matériel végétal fourni serait ainsi plus vigoureux, donc dans les meilleurs catégories des normes de classement.

- le sélectionneur qui utilise des résultats juvéniles qu'il s'agisse des plantations comparatives de provenances ou de descendances, doit également mettre ses plants hors concurrence. Dans la négative, des biais importants peuvent être apportés dans les classements effectués. En effet, des populations vigoureuses peuvent se trouver pénalisées dans leurs performances pour l'effet de la densité, alors que les moins vigoureuses ne le seraient pas. La densité peut également fortement perturber la phénologie des populations étudiées.

Compte tenu de ces résultats, il conviendrait de réviser les écartements généralement adoptés au repiquage.

Reçu pэur publication en juin 1972.

\section{REMERCIEMENTS}

MM. Jacquard, du Centre d'Études phytosociologiques et écologiques de Montpellier, et Decourt, de la Station de Sylviculture et Production du Centre national de Recherches forestières, ont bien voulu accejter de relire le manuscrit; leurs conseils ont contribué à sa mise en formes; qu'ils trouvent ici l'expression de mes sincères remerciements.

\section{SUMMARY}

DOUGLAS FIR SEEDLINGS DENSITY, COMPETITION EFFECTS AND INTRASPECIFIC VARIATION IN NURSERY

- Generally speaking the question of the competition through the density between the individuals within a population is approached without taking into consideration of the genetic characteristics. However genotype density interactions may be existing. This paper presents the results of an experiment devoted to the study of the intraspecific variation against the density of young Douglas Fir seedlings from few provenances. 
- The study was led on 3 provenances ( 2 from America, 1 artificial from France). The seeds were sowed directly in seed-bed (not later lining out) with the following densities : $400 / \mathrm{m}^{2}(5 \times 5 \mathrm{~cm})$, $100 / \mathrm{m}^{2}(10 \times 10 \mathrm{~cm})$ and $25 / \mathrm{m}^{2}(20 \times 20 \mathrm{~cm})$. The design is a factorial design $(3$ provenances $\times 3$ densities) with complete blocks (4 replications). Various phenologic and vigour characters were assessed.

- It appears that the effect of the density is negative on the mean diameter (recognized result) but also early on the mean height. But interactions provenance density are not really significant.

- The results of the study for the characters variation (distribution, standard deviation and coefficient of variation) are :

- clear effect of the density at least on the diameter : asymetric distribution on the left, positive correlation between the coefficient of variation and the density.

- not interactions provenance $x$ density.

- The negative effect of the density on the branch growth is clear. At an individual level, it appears that the branch growth varies in direct ratio to the leader-shoot growth. The variation law is the same for the 3 provenances however great the density.

- The density has the same effect upon the lamma shoot formation as upon the vigour. The terminal bud flushing seems to be subordinated to the seedling height and to the fertility in broad sense, themselves subordinated to the density.

- With regard to the provenances tests in the nursery, the results are showing it would be necessary for the tester to use lower densities than usualy. Without this care, the populations ranging may be affected. On this small provenances sample, it does not appear interactions provenance $x$ density, but these results cannot be extrapolated to the whole the Douglas fir species.

\title{
ZUSAMMENFASSUNG
}

\author{
POPULATIONSDICHTE, KONKURRENZEFFEKTE UND INTRASPEZIFISCHE VARIABILITAT BEI
}

\author{
DOUGLASIEN EINER BAUMSCHULE
}

Die Konkurrenzprobleme im Zusammenhang mit der Populationsdichte (Pflanzanabstand) wurden meist unter Ausserachtlassung der genetischen Populationsmerkmale untersucht. Es ist jedoch anzunehmen, dass Wechselwirkungen zwischen Genotyp und Dichte bestehen. Die vorliegende Arbeit bringt die Ergebnisse eines Versuches, der die Zusammenhänge zwischen der intraspezifischen Variabilitât und der Populationsdichte von jungen Douglasienpflanzen verschiedener Herkunft bestimmen soll.

Der Versuch wurde mit drei Douglasienherkünften (zwei amerikanische und eine künstliche französische Herkunft) durchgeführt. Die Samen wurden direkt (ohne Verschulung) in den Abständen $5 \times 5 \mathrm{~cm}\left(400 / \mathrm{m}^{2}\right), 10 \times 10\left(100 / \mathrm{m}^{2}\right)$ und $20 \times 20 \mathrm{~cm}\left(25 / \mathrm{m}^{2}\right)$ eingebracht. Der Versuchsplan ist faktoriel (3 Herkünfte $\times 3$ Dichten) in vollständigen Blöcken (4 Wiederholungen) angelegt.

Verschiedene phänologische Merkmale und Wachstumsgrössen wurden gemessen.

Die Dichte hat eine depressiere Wirkung nicht nur auf das Durchmessermittel (klassisches Ergebnis) sondern auch auf die Mittelhöhe und zwar sehr frühzeitig. Es zeigt sicher keine deutlich signifikante Wechselwirkung Herkunft $\times$ Dichte.

Die Untersuchung der Variabilität der Meıkmale (Verteilung, Standard-abweichung, Variationskœffizient) zeigt, dass der Dichteeffekt zu einer ziemlich deutlichen Konkurrenz zumindest beim Durchmesser führt (linksschiefe Verteilung, positive Beziehung zwischen Variationskœffizient und Dichte). Es zeigt sich keine signifikante Wechselwirkung Herkunft $\times$ Dichte.

Die depressive Wirkung der Dichte auf die Astentwicklung ist sehr deutlich. Auf individueller Ebene zeigt sich, dass die Astentwicklung der Endtriebentwicklung proportional ist, und, unabhängig von Herkunft und Dichte, dieselbe Gesetzmässigkeit aufweist.

Bei den phänologischen Merkmalen zeigt sich, dass die Johannistriebbildung im gleichen Sinne wie die Wuchskraft betroffen wird. Das Austreiben ist sowohl durch die Höhe der Pflanzen als auch durch die Saatbettgüte im weitesten Sinne (Nährztoff-Licht-u. Wasserhaushalt) bedingt, welche wiederum von der Dichte abhängen. 
Die begrenzte Anzahl von Herkünften erlaubte es nicht, eine Wechselwirkung Herkunft $\times$ Dichte festzustellen. Die Ergebnisse können daher nicht auf andere Douglasienherkünfte extrapoliert werden.

Bezüglich der Herkunftsprüfung im Pflanzgarten scheint es jedoch notwendig, weitere als die bisher üblichen Pflanzabstände zu wählen, um die Konkurrenz auszuschalten. Ohne diese Vorsichtsmassregel läuft man Gefahr zumindest die wüchsigsten Herkünfte falsch einzustufen (Anderung der Mittelwerte und vor allem der Varianzen). Dies ist beim Merkmal « Austreiben » in erhöhtem Masse der Fall.

\section{RÉFÉRENCES BIBLIOGRAPHIQUES}

Bartoli P., Decourt N., 1971 Densité de plantation. Étude bibliographique et premiers résultats d'une expérience sur le Douglas (Pseudotsuga menziesii Mirb.). Ann. Sci.forest., 28 (1), 59-81.

Delvaux J., 1966 Contribution à l'étude de l'éducation des peuplements. II. A propos de la distribution de fréquences de diamètres et de hauteurs. III. Essais préliminaires à l'étude du facteur compétition. IV. La compétition au niveau des classes sociales. Trav. Stat. Rech. Groenendaal, 33, 7-59.

Delvaux J., 1971 Contribution à l'étude de l'éducation des peuplements X. Compétition interindividuelle chez le Douglas (Pseudotsuga menziesii Mirb, Franco var menziesii). Trav. Stat. Rech. Groenendaal, B 36, 7-23.

FAlCONER D. S., 1960 Introduction to quantitative genetics. Oliver and Boyd, Edinburgh and London, 365 p.

Illy G., LemorNe B., 1970 Densité de peuplement, concurrence et coopération chez le Pin maritime. I Premiers résultats d'une plantation à espacement variable. Ann. Sci. forest., 27 (2), 127-155.

KoyAma H., KIRA T., 1956 Intraspecific competition among higher plants. VIII - Frequency distribution of individual plant weight as affected by the interaction between plants. J. Inst. Polytechn., Osaka City Univ. Ser. D 7, 79-94.

Lacaze J.-F., Tomassone R., 1967 Contribution à l'étude de la variabilité infraspécifique du Douglas (Pseudotsuga menziesii Mirb.) Ann. Sci. forest., 24 (1), 85-106.

LACAze J.-F., 1969 Étude de la variabilité infraspécifique de l’Épicéa (Picea abies Karst.) — Provenances françaises et polonaises. Résultats au stade juvénile. Ann. Sci. forest., 26 (3), 345-396.

LaCaze J.-F., Arbez M., 1971 Variabilité infraspécifique de l'Épicéa (Picea abies Karst., partie septentrionale de l'aire française). Héritabilité et corrélations génétiques de quelques caractères au stade juvénile. Ann. Sci. forest., 28 (2), 141-183.

Walters J., KozaK A., 1967 The effect of chemical fertilisation on the formation of lammas shoots in Douglas fir. Res. Pap. from the files of the Faculty of Forestry, U.B.C. Vancouver, Canada $\mathrm{n}^{\circ} 76$, mars 1967.

Walters J., Soos J., 1961 Some observations on the relationsship of lammas shoots to the form and growth of Douglas fir seedling. Res. Pap. from the files of the Faculty of Forestry, U.B.C. Vancouver Canada, $n^{\circ} 40$, mars 1961. 